AORTA AND

PERIPHERAL

CIRCULATION

\title{
The year in cardiology: aorta and peripheral circulation The year in cardiology 2019
}

Christian Heiss",\#, Alex Pitchert, Jill J.F. Belch ${ }^{\ddagger}$, Marco De CarloJ, Holger Reinecke ${ }^{\infty}$, Iris Baumgartner ${ }^{\S}$, Lucia Mazzolai ${ }^{\ominus}$ and Victor Aboyans ${ }^{\Delta, * * *}$ on behalf of the ESC Working Group

SAHeart 2020;17:172-182 on Aorta and Peripheral Vascular Diseases

\author{
*Department of Clinical and Experimental Medicine, University of \\ Surrey, Stag Hill, Guildford, United Kingdom \\ "Surrey and Sussex Healthcare NHS Trust, East Surrey Hospital, \\ Redhill, United Kingdom \\ tDivision of Cardiovascular Medicine, Radcliffe Department of \\ Medicine, University of Oxford, John Radcliffe Hospital, Headington, \\ Oxford, United Kingdom \\ 抽e Institute of Cardiovascular Research, University of Dundee, \\ Ninewells Hospital and Medical School, Ninewells Hospital, \\ Dundee, United Kingdom \\ 'Cardiac Catheterisation Laboratory, Cardiothoracic and Vascular \\ Department, Azienda Ospedaliero-Universitaria Pisana - Cisanello, \\ Pisa, Italy \\ ${ }^{\infty}$ Department of Cardiology, University Hospital Muenster, \\ Muenster, Germany \\ ${ }^{\S}$ Clinical and Interventional Angiology, Swiss Cardiovascular Center \\ Bern, Insel Group AG, University Hospital Bern, Bern, Switzerland \\ Division of Angiology, Department of Heart and Vessel, Lausanne \\ University Hospital, Lausanne, Switzerland \\ $\triangle$ Department of Cardiology, Dupuytren University Hospital, \\ Limoges, France \\ "*nserm 1094, Limoges School of Medicine, Limoges, France
}

Address for correspondence:

Prof Christian Heiss

Department of Clinical and Experimental Medicine

University of Surrey

Stag Hill I4AY04

Guildford GU2 7XH

United Kingdom

Email:

c.heiss@surrey.ac.uk

\section{INTRODUCTION}

Like previous years, ${ }^{(1-3)}$ the current article reviews groundbreaking science published in 2019 in the area of aortic and peripheral arterial diseases (PAD), as well as venous thromboembolic disease (VTE), which will affect our daily clinical practice. With the growing recognition of PAD, it will be necessary to consolidate imprecisions in terminology. Many are used to the acronym PAD for atherosclerotic disease of the lower extremity arteries. Others have used the same acronym to qualify atherosclerotic disease of the lower extremity arteries and carotid arteries. In the current article and in line with the European Society of Cardiology (ESC) guidelines, ${ }^{(4)}$ we have stringently used the specific terms lower extremity arterial disease (LEAD) and have reserved PAD as the umbrella term encompassing all arterial diseases other than relating to the aorta and coronaries.

\section{VASCULAR BIOLOGY/TRANSLATIONAL RESEARCH}

The extent to which genetic factors contribute to PAD development and if they are shared or distinct between LEAD, cerebral, and coronary arteries are largely unknown. In a genome-wide association study in the Million Veteran Program, $\sim 32$ million DNA sequence variants were tested for PAD (31 307 cases, 2 II 753 controls) and combined with electronic health records. ${ }^{(5)}$ The results were replicated in an independent sample from the UK Biobank. They identified 19 LEAD loci ( 8 not previously reported): I I loci were associated with disease in 3 vascular beds (coronary, cerebral, and lower extremity), including LDLR, LPL, and Lp(a) (Figure I); 4 loci appeared to be specific for LEAD, including F5 p.R506Q (Factor $V$ Leiden variant), highlighting the pathogenic role of thrombosis in LEAD and supporting Factor $\mathrm{Xa}$ inhibition as a therapeutic strategy.

Despite the fact that numerous long non-coding RNAs (IncRNA) have been identified, only a few of them have been studied with respect to endothelial cell homeostasis or vascular disease development. One of them, the pro-angiogenic IncRNA MANTIS, may be clinically relevant in carotid disease. ${ }^{(6)}$ In fact, the protective effects of laminar flow and statins are, at least in part, attributed to the expression of MANTIS. The mechanisms involve epigenetic rearrangements and the transcription factors Krüppel-like factor 2 and 4. As induction of MANTIS mimics the beneficial effects of statins on endothelial function, the authors proposed that strategies to increase MANTIS might improve vascular function in patients not responding to statin therapy. 
The transcriptional activity of nuclear receptors that regulate key pathophysiological processes in atherosclerosis development is controlled by the nuclear receptor corepressors (NCOR), scaffolding proteins that form the basis of large corepressor complexes. Oppi, et al.(7) investigated the role of NCORI in atherogenesis. Myeloid cell-specific deletion of NCORI in LDL receptor knockout mice aggravated atherosclerosis development. Macrophage NCORI-deficiency led to increased foam cell formation, enhanced expression of proinflammatory cytokines, and atherosclerotic lesions characterised by larger necrotic cores and thinner fibrous caps. The immunometabolic effects of NCORI were mediated via suppression of peroxisome proliferator-activated receptor gamma (PPAR $\gamma$ ) target genes in mouse and human macrophages, which lead to an enhanced expression of the CD36 scavenger receptor and subsequent increase in oxidised LDL uptake in the absence of NCORI. Interestingly, in human atherosclerotic plaques, the expression of NCORI was reduced, whereas the PPAR $\gamma$ signature was increased, and this signature was more pronounced in ruptured compared with non-ruptured carotid plaques. The data suggest that stabilising the NCORI-PPAR $\gamma$ binding could be a promising strategy to block the pro-

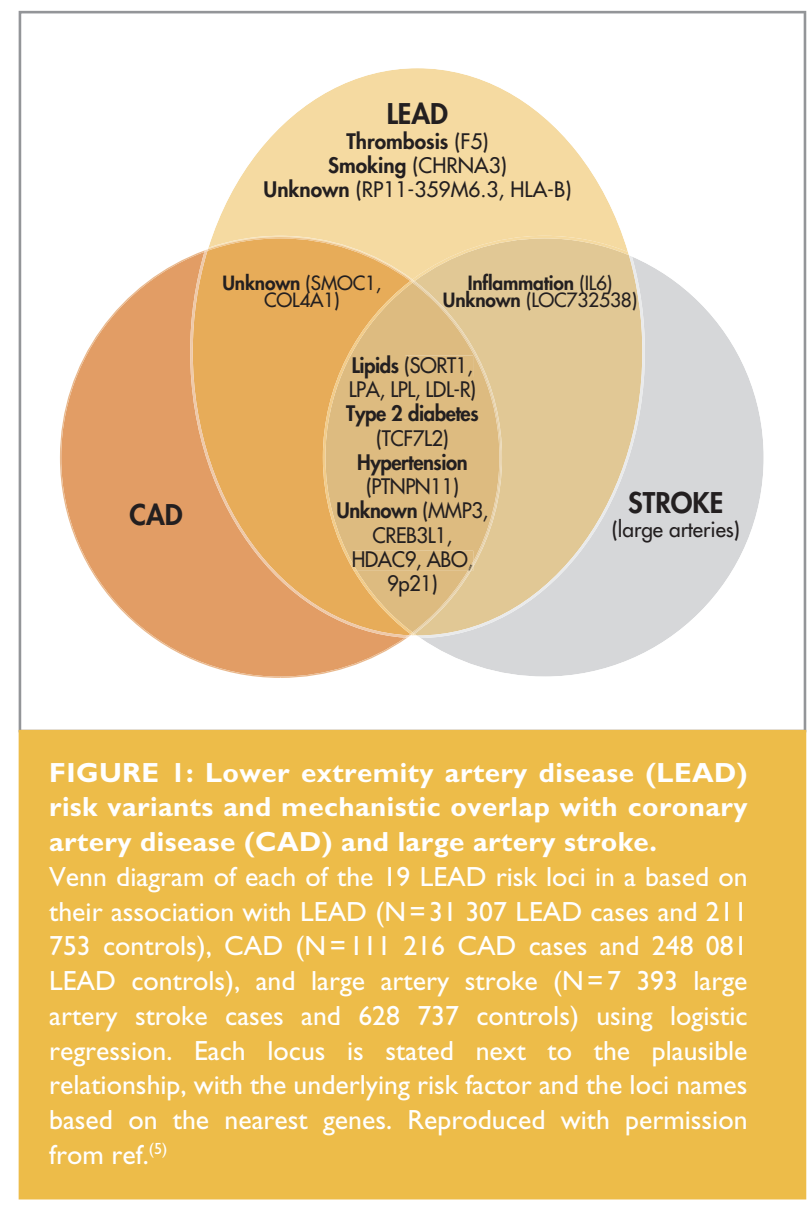

atherogenic functions of plaque macrophages and lesion progression.

Radiotherapy-induced cardiovascular disease (CVD) is an emerging problem in a growing population of cancer survivors where traditional vascular treatments have limited benefits. Using a translational approach, it was now shown that human irradiated blood vessels exhibit elevated levels of inflammation signals associated with inflammasome activation long after radiotherapy, and similar changes occurred in a mouse model of localised irradiation to the heart and carotids. ${ }^{\left({ }^{8}\right)}$ In the model, the localised inflammatory response was ameliorated by an interleukin (IL)- I receptor antagonist. Clinical studies in humans now need to evaluate IL-I blockade as a potential treatment of radiotherapy-induced CVD.

Subjects with $L p(a)$ elevation have increased arterial wall inflammation and cardiovascular risk. Stiekema, et al. ${ }^{(9)}$ evaluated whether evolocumab, which as opposed to statins lowers both LDL-cholesterol and Lp(a), attenuates arterial wall inflammation in the index vessel (carotid or thoracic aorta) in patients with elevated $L p(a)(>200 \mathrm{mg} / \mathrm{dL})$. In this multicentre, randomised, double-blind, placebo-controlled study, 129 patients were randomised to monthly subcutaneous evolocumab $420 \mathrm{mg}$ or placebo. Compared with placebo, evolocumab reduced LDLcholesterol by $60.7 \%$ [95\% confidence interval $(\mathrm{Cl}) 65.8$ - 55.5] and $\operatorname{Lp}(\mathrm{a})$ by only $13.8 \%(95 \% \mathrm{Cl} 19.3$ - 8.5). Importantly, arterial wall inflammation [assessed by [(positron emission tomography with 2-deoxy-2-[fluorine-18]-fluoro-D-glucose integrated with computed tomography)] ${ }^{18} \mathrm{~F}-\mathrm{FDG}$ PET/CT] was not significantly altered with evolocumab at Week 16. This supports that, beyond economic issues, statins remain the first pillar of lipid-lowering therapies, which ties in with current lipid guidelines. ${ }^{(10)}$

Another large study characterised serum metabolic signatures associated with atherosclerosis in the carotid and coronary arteries and subsequently their association with incident CVD among 3867 participants from the Multi-Ethnic Study of Atherosclerosis (MESA), with replication among 3569 participants from the Rotterdam and LOLIPOP studies.( ${ }^{(1)}$ They showed that $30 \mathrm{IH}$ NMR (proton nuclear magnetic resonance spectroscopy) measured metabolites were associated with coronary artery calcium and/or carotid intima-media thickness. Metabolites associated with atherosclerosis were largely consistent between the carotid and coronary vascular beds and predominantly tag pathways that overlap with the known cardiovascular risk factors: disturbances in lipid and carbohydrate metabolism, branched chain, and aromatic amino acid metabolism, as well as oxidative stress and inflammatory pathways. 


\section{VASCULAR BIOMARKERS AND CARDIOVASCULAR RISK}

Multimodality vascular assessment enables us to evaluate the atherosclerotic process and the cardiovascular risk. In a population-based study(12) using hybrid ${ }^{18} \mathrm{~F}-\mathrm{FDG}$ PET and magnetic resonance imaging (MRI), arterial inflammation was detected in $48 \%$ of participants of 40 - 54 years of age, increasing steadily by the number of risk factors. Aortic, carotid, and/or iliofemoral plaques were present in $90 \%$ of cases, but most inflammation was depicted in the plaque-free zones. Inflammation was present only in 11\% of plaques, suggesting arterial inflammation in the early stage of the atherosclerosis process. An experimental study went one step further and developed an integrative multiparametric PET/MRI protocol that allows non-invasive assessment of different processes relevant to atherosclerosis progression. ${ }^{(13)}$ Using clinically approved nanobody radiotracers, they were able to study different biomarkers of atherosclerosis progression, namely vascular cell adhesion molecule-I, lectin-like oxidised lowdensity lipoprotein receptor-I, and macrophage mannose receptor, which correlated with histopathological findings in mice and rabbits.

Atherosclerosis is even identifiable in adolescence, especially in the case of an unhealthy lifestyle: in an observational study ${ }^{(14)}$ including I 266 young participants aged 13 - 17 years, aortic stiffness, estimated by carotid-femoral pulse-wave velocity (cfPWV) was proportionally increased by the tobacco smoking and alcohol drinking intensities, with a strong potentiation when both were combined (Figure 2). At these ages, smoking and drinking cessation lead to normalisation of cfPWV. In the other lifespan tip, vascular markers could be useful to downgrade the estimated risk in elderly people who would have an indication for statins based on risk scores, highly affected by age. In a cohort of 5805 healthy elderly participants (mean age 69 years), normal $(<10)$ coronary calcium score and no carotid plaque on ultrasound were the most
A

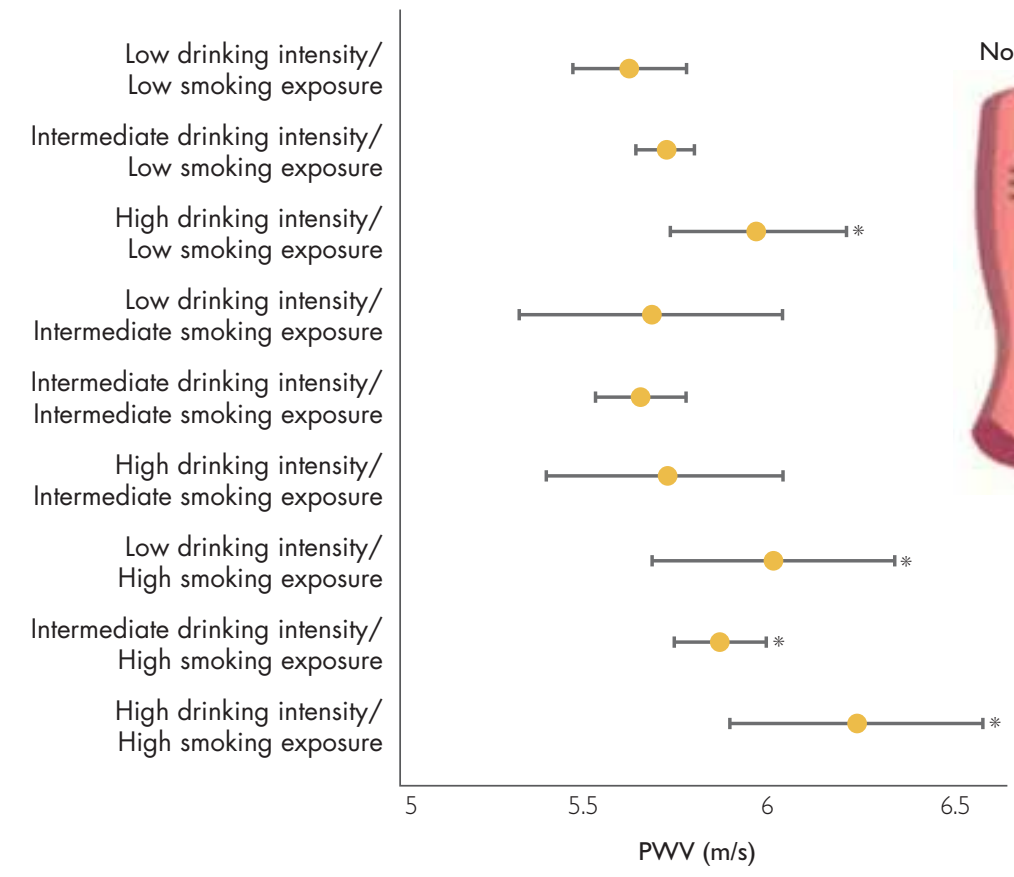

adjusted for gender, SBP, BMI, LDL, SES, CRP
B
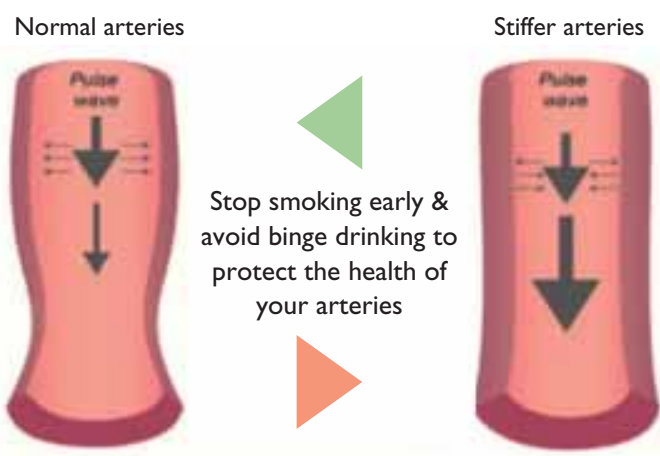

Accelerated

atherosclerosis

Unhealthy behaviours in adolescents

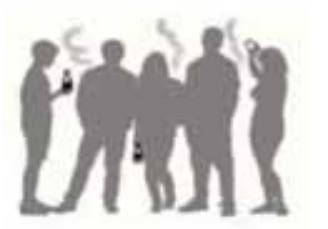

Drinking and cigarette smoking

FIGURE 2: (A) The combined effect of smoking over a lifetime and intensity of drinking on arterial stiffness.

The combination of high-intensity drinking with lifetime smoking exposure is shown. Pulse wave velocity measurements are expressed as mean values and $95 \%$ confidence intervals around the mean on the x-axis. The participants who had "high" drinking intensity and "high" smoking exposure had the highest pulse wave velocity compared with the "low lifetime smoking exposure" and "low drinking intensity" *p $<0.05$

(B) Unhealthy behaviours in adolescents (drinking and cigarette smoking) are associated with increased carotid to femoral pulse wave velocity (stiffer arteries) and accelerated atherosclerosis.

Stopping smoking in adolescents and reducing binge drinking has potential for reversibility of arterial stiffening.

Reproduced with permission from ref. ${ }^{(14)}$ 
powerful vascular markers to downgrade the predicted cardiovascular risk, with respective net reclassification index of 0.29 and 0.14 , avoiding unnecessary statin prescription in $34 \%$ and $21 \%$ of cases. ${ }^{(15)}$

Ultrasound vascular imaging can efficiently improve patients' adherence to medical advice for healthy lifestyle. The visualisation of asymptomatic atherosclerotic disease for optimum cardiovascular prevention (VIPVIZA) open controlled trial ${ }^{(16)}$ randomised 3532 individuals $>40$ years attending a screening programme; the control group received regular risk factors' screening and guidelines-based management but the results of carotid ultrasound were not disclosed, while the intervention group received, along with their general practitioners, a pictorial presentation of the carotid ultrasound results, including colourscaled presentations of vascular age based on intima-media thickness, and plaque identification. A nurse was called 2 - 4 weeks later to reassure participants and provide any information needed. The same pictorial information was repeated after 6 months. The baseline Framingham risk score (FRS) and SCORE were respectively at 12.9 and 1.28. At I year, both scores were significantly lower in the intervention group (-1.07, $p=0.0017$ for FRS and -0.16, $p=0.001$ for SCORE), with more striking results in the high-risk group $(-2.16$ and -2.85 , respectively). The persistence of these results and their consequences on CVD events need further evaluation.

\section{CEREBROVASCULAR DISEASE}

Excessive arterial pulsatility may contribute to cognitive decline and risk of dementia via damage to the fragile cerebral microcirculation. As part of the Whitehall II study, (17) peak forward-travelling compression wave intensity (FCWI) was assessed using Duplex ultrasound within the common carotid arteries in 3191 individuals (mean age $=61$ years; $75 \%$ male) and serial measures of cognitive function were taken at baseline and almost 10 years later. Higher FCWI at baseline was associated with accelerated cognitive decline during follow-up and this association was largely driven by cognitive changes in individuals with the highest FCWI. Compared to other participants, this group was approximately $50 \%$ more likely to exhibit cognitive decline, even after adjustments for multiple potential confounding factors.

While intensive lipid lowering is recommended after transient ischaemic attack (TIA) and ischaemic stroke, the target level for LDL to reduce cardiovascular events after stroke has not been well studied. In a parallel group trial, 2860 patients with recent ischaemic stroke or TIA and evidence for cerebrovascular and coronary artery atherosclerosis were randomised to either LDL target of $<70 \mathrm{mg} / \mathrm{dL}$ or 90 - I I $0 \mathrm{mg} / \mathrm{dL}$ with a statin, ezetimibe, or both.(18) During a mean follow-up of 3.5 years, major cardiovascular events occurred less in the lower target group [8.5\% vs. 10.9\%; hazard ratio (HR) 0.78 (95\% Cl 0.61 0.98)].

Patients with high stroke risk and atrial fibrillation who are unsuitable for oral anticoagulants (OACs) require alternative stroke prevention strategies. The multicentre, non-randomised, first-in-human clinical Carotid Artery Implant for Trapping Upstream Emboli for Preventing Stroke in Atrial Fibrillation Patients (CAPTURE) trial sought to determine the feasibility and safety of a novel permanent coil filter directly placed into both common carotid arteries, and designed to capture emboli $>1.4 \mathrm{~mm}$ in diameter. ${ }^{(19)}$ Patients received aspirin/clopidogrel for 3 months, and aspirin thereafter. In 3 centres, 25 patients with atrial fibrillation, with $\mathrm{CHA}_{2} \mathrm{DS}_{2}-\mathrm{VASc} \geq 2$, who were unsuitable for OACs and had no carotid stenosis $>30 \%$ were enrolled. The procedure success was 92\%; I patient had unilateral deployment. There were no device/procedure-related major adverse events. After 6-month mean follow-up, asymptomatic thrombi were detected in 4 patients (I bilateral, 4 unilateral) and the thrombi dissolved with subcutaneous heparin. Permanent carotid filter placement for stroke prophylaxis seems technically feasible and safe. Larger studies and a comparison with the use of left atrial appendage occluders are necessary.

\section{AORTIC DISEASE}

A common challenge in the emergency room is to distinguish patients with symptoms suggestive of acute aortic syndrome (AAS) requiring a computed tomography scan, from others. In a study of 839 patients attending the emergency room with suspected AAS, focused cardiac ultrasound, integrated into a strategy including clinical assessment and (for low-risk patients) $D$-Dimer testing, enabled the correct identification of all patients with aortic dissection (AD), although the upper border of the $95 \% \mathrm{Cl}$ was $1.2 \%{ }^{(20)}$ These findings confirm the importance of transthoracic chocardiography in the diagnostic strategy of AAS as suggested in the last ESC guidelines. (21)

The term AAS has become commonplace, but constitutes a range of disease entities, which may not have the same pathophysiological mechanisms, responses to treatment or outlook. Among I 012 patients, those with intra-mural haematoma $(\mathrm{MH})(\mathrm{n}=340)$ had a much better short- and long-term mortality than those patients with $A D(n=672){ }^{(22)}$ Taking the Type B IMH in-hospital mortality, estimated at 1.5\%, as reference, the overall crude in-hospital mortality of Type A AD 
was $15.0 \%$ with an adjusted hazard ratio (aHR) of 30.4, compared to Type A IMH mortality of 8.0\% (aHR 4.85) and Type B AD mortality of $5.0 \%$ (aHR $3.5 \mathrm{I}$ ).

Identifying patients with Marfan syndrome at particular risk of $A D$ is currently based on the absolute diameter of the aorta, the growth rate, and the presence/absence of a family history of AD. A novel additional approach may be the evaluation of the longitudinal strain of the proximal aorta by MRI (Figure 3A).(23) Higher strain rates were associated with more rapid aortic expansion and appeared to predict clinical outcomes. If proximal aortic strain is reproducible and if these findings are replicated in larger cohorts, this may help to inform the need for, and timing of, surgery in these patients.

Complex flow patterns are identified in the true and often the false lumen after AD. A better understanding of these flow dynamics may explain the differing behaviour of ADs over the long term. Recent insights into this process have become available from a detailed study categorising flow patterns using echo Doppler. The potential for prognostic implications is discussed (Figure 3B). (24)

The optimal management of descending thoracic aortic aneurysms is controversial. A retrospective study on a propensity-adjusted population of Medicare beneficiaries found lower peri-operative and overall mortality in patients undergoing thoracic endovascular aortic repair (TEVAR) compared to open repair, but with a higher risk of reintervention. ${ }^{(25)}$ The odds of peri-operative mortality were greater for open surgical repair and depended on the centre volume: high-volume centre, odds ratio (OR) $1.97(95 \% \mathrm{Cl} 1.5$ - 2.6); low-volume centre, OR 3.62 (95\% Cl 2.9 - 4.5). The restricted mean survival time difference favoured TEVAR at 9 years, -209 days ( $95 \%$ Cl -299 to -120 days) for open surgical repair. The risk of reintervention was lower for open surgical repair, HR 0.40 (95\% Cl $0.34-0.60)$.

In the last ESC guidelines on the management of aortic diseases, ${ }^{(21)}$ both open surgery and endovascular aneurysm repair (EVAR) of abdominal aneurysms received Class I recommendation, based on several head-to-head trials enrolling patients with suitable anatomy for both options. While, in the short term, EVAR was associated with lower mortality, this difference was gradually annihilated over time, while in turn, EVAR requested repeated $X$-ray exposure and reinterventions for endoleaks. The results of very long-term follow-up (I4 years) of the Open vs. Endovascular Repair (OVER) trial(26) are interesting in that they show no mortality or secondary procedure difference beyond the first years. These results support current recommendations; importantly, mortality was largely not aneurysm-related (only $2.7 \%$, mostly postoperative), and mostly due to cardiovascular causes, emphasising the need for maximal preventive measures in these patients. Finally, gender-specific evidence is still lacking, as women constituted $<10 \%$ of all participants.

\section{LOWER EXTREMITY ARTERY DISEASE}

Lower extremity arterial disease is an increasing public health problem according to the latest global epidemiology report. ${ }^{(27)}$ In 20I0, LEAD, defined as $A B \mid \leq 0.9$, affected 202 million subjects worldwide; this number increased by $22 \%$ to 237 million in 2015. The overall prevalence in subjects aged $\geq 25$ years was $5.6 \%(95 \% \mathrm{Cl} 3.8-8.6)$, and higher in highincome countries than in low- and middle-income countries (LMIC) (7.4\% vs. 5.1\%), although the vast majority of patients (73\%) lived in LMIC. This prevalence was similar between sexes, with higher rates of young ( $<50$ years) patients in LMIC.

The association of LEAD with major adverse cardiovascular events (MACE) is well documented, whereas its association with limb events is less clear. In the Veterans Aging Cohort Study, including 125674 subjects without history of prior amputation, the incidence of amputation over a median of 9.3 years of follow-up was 1.2 per I 000 person-years. (28) The presence of LEAD conferred a 13.9-fold increase in amputation risk, but microvascular disease (MVD), defined as retino-, neuro-, and/or nephropathy, was also associated with a 3.7fold risk increase, and the combination of LEAD and MVD lead to a 22.7-fold increased risk. Importantly, MVD alone was associated with $15 \%$ of all below-the-knee amputations.

Among the 13885 patients with an $A B \mid \leq 0.8$ or prior lower extremity revascularisation (LER) randomised to ticagrelor vs. clopidogrel in the Examining Use of Ticagrelor in Peripheral Artery Disease (EUCLID) trial, the rate of acute limb ischaemia (ALI) requiring hospitalisation was 0.8 per 100 patient-years, with no difference between treatment arms. (29) Acute limb ischaemia was strongly associated with subsequent MACE (aHR 1.4, 95\% Cl 1.0 - 2.1), all-cause mortality (aHR 3.3, 95\% $\mathrm{Cl} 2.4$ - 4.6), and major amputation (aHR 34.2, 95\% Cl 9.7 20.8). Previous LER, baseline atrial fibrillation, and baseline $A B I \leq 0.60$ were independent predictors of ALI. In this trial, a second analysis showed that $12.5 \%$ of patients experienced LER during the trial. ${ }^{(30)}$ Independent predictors of post-randomisation LER were prior history and type of prior LER $(p<0.000 \mathrm{I})$, living in North America or Europe $(p<0.000 \mathrm{I})$, presence of limb symptoms at baseline (HR 1.3; 95\% Cl 1.2 I.5), diabetes (HR I.3; 95\% Cl I.I - 1.4), and smoking (HR I.2; 
95\% Cl 1.1 - 1.4). Cardiac and limb events were numerically higher in patients undergoing surgical procedures, but surgical patients experienced fewer LERs after the index LER.

A growing proportion of ALI hospitalisations occurs in cancer patients who experience arterial thromboembolism. In the population-based Surveillance Epidemiology and End ResultsMedicare linked dataset, 374331 patients $\geq 67$ years with primary diagnosis of breast, lung, prostate, colorectal, bladder, uterine, pancreatic and gastric cancer, or non-Hodgkin lymphoma, were identified. ${ }^{(31)}$ The risk of arterial thromboembolic events began to increase 150 days before the date of cancer diagnosis in older patients and peaked in the 30 days before cancer diagnosis, when $0.62 \%$ of patients suffered an arterial thromboembolic event vs. $0.11 \%$ in control subjects (OR 5.63; $95 \%$ Cl 5.07 - 6.25).

Lipid lowering is a key element of LEAD treatment. ${ }^{(4)}$ The 2019 ESC guidelines recommend a LDL-cholesterol reduction of $\geq 50 \%$ and a goal of $<55 \mathrm{mg} / \mathrm{dL}(1.4 \mathrm{mmol} / \mathrm{L})$ for LEAD patients, to be achieved with statins, plus ezetimibe and PCSK9 inhibitors if needed. ${ }^{(10)}$ A recent pre-specified analysis of the Evaluation of CV Outcomes After an Acute Coronary Syndrome During Treatment With Alirocumab (ODYSSEY

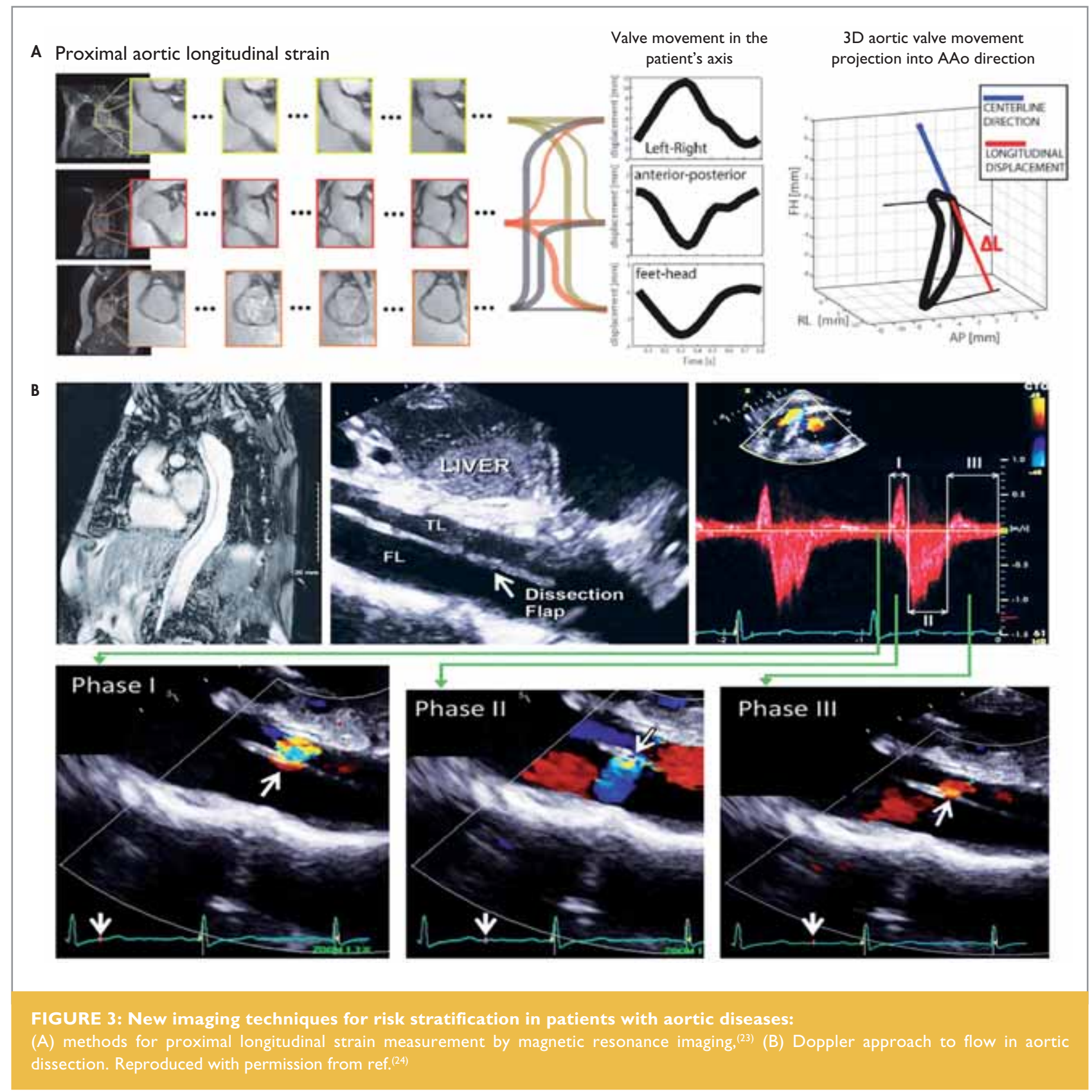


OUTCOMES) trial further supports these recommendations. ${ }^{(32)}$ After a median follow-up of 2.8 years, the I 554 patients with atherosclerotic disease in 2 or 3 vascular beds (coronary, lower limb, and/or cerebrovascular) showed a significantly larger absolute risk reduction with alirocumab, as compared to patients with isolated coronary artery disease (CAD). The absolute risk reduction regarding MACE was 1.9\% (95\% Cl -2.4\% - 6.2\%) and 13.0\% (95\% Cl-2.0\% - 28.0\%) for 2 and 3 vascular beds, respectively, whereas regarding all-cause mortality it was $1.3 \%(95 \% \mathrm{Cl}-1.8 \%-4.3 \%)$ and $16.2 \%(95 \%$ Cl $5.5-26.8 \%)$, respectively.

Another pillar of the medical treatment of LEAD is optimal control of arterial hypertension. ${ }^{(4,33)}$ A recent analysis from the Atherosclerosis Risk in Communities (ARIC) study evaluated the impact of different stages of hypertension on the development of LEAD. ${ }^{(34)}$ During a median follow-up of 25.4 years, a systolic blood pressure (BP) $\geq 140 \mathrm{mmHg}$ or diastolic BP $\geq 90 \mathrm{mmHg}$ was associated with a higher rate of incident LEAD diagnosis (HR 2.40; 95\% Cl 1.72 - 3.34), independent of the use of anti-hypertensive medications. Higher BP categories showed significant associations with incident LEAD starting from 120 - $129 \mathrm{mmHg}$ for systolic BP and $\geq 90 \mathrm{mmHg}$ for diastolic BP. These data emphasise the need for BP control to prevent the development of LEAD.

While supervised exercise training is a mainstay of the management of claudication, ${ }^{(4)}$ low adherence rates limit its clinical application. In a randomised study, I 56 participants were allocated to supervised treadmill exercise, supervised resistance training, or oral advice about nutrition and training.(35) After 6 months, the 6 minute walk distance improved only in the treadmill exercise group $(36.1 \mathrm{~m}, 95 \% \mathrm{Cl} \mid 3.9-58.3)$, but at 12 months neither treadmill nor resistance significantly differed from baseline or control (walking distance: $+7.5 \mathrm{~m}$ and $+6.1 \mathrm{~m}$ ). These results highlight the need for long-term supervised exercise programmes to maintain benefits. Additionally, a systematic review of 84 studies reported that alternative training modalities (circuit exercise, low-pain and pain-free walking, resistance training, upper/lower limb ergometry, and pole striding) had significantly higher adherence and completion rates vs. traditional exercise training $(85.5 \%$ vs. $77.6 \%$, and $86.6 \%$ vs. $80.8 \%$, respectively). ${ }^{(36)}$

With lack of randomised controlled trials (RCTs), a large gap in evidence regards the best revascularisation strategy in chronic limb threatening ischaemia (CLTI). In a retrospective analysis, 16800 patients with CLTI who had first surgical LER (36\%) were compared to those with first endovascular LER (64\%). ${ }^{(37)}$ The endovascular group was younger, but suffered from more comorbidities, including renal failure (36\% vs. 24\%), CAD (34\% vs. $32 \%$ ), heart failure (19\% vs. 15\%), and diabetes (65\% vs. $58 \%$; all $p<0.05)$. In a propensity-matched analysis, a surgery-first strategy was associated with worse amputationfree survival (HR 1.16,95\% Cl 1.13 - 1.20), while an endovascular-first strategy was associated with higher reintervention rates (HR 1.19,95\% Cl 1.14 - 1.23) after 80 months of follow-up. Mortality was similar between groups (HR 0.94, $95 \% \mathrm{Cl} 0.89-1.11$ ). These results suggest that an endovascularfirst approach might be preferable regarding amputation-free survival.

Several trials have shown the superiority of drug-eluting stents (DES) and drug-coated balloons (DCBs) vs. plain balloon angioplasty (PTA) in patients with femoropopliteal disease. ${ }^{(1-3)}$ The 5-year results of the IN.PACT SFA trial showed the persistence of clinical benefits, with $74.5 \%$ freedom from clinically driven target lesion revascularisation with DCBs vs. $65.3 \%$ with PTA $(p=0.020)$, although this benefit was non-significant in diabetics $(70.3$ vs. $64.4 \%, p=0.24) .{ }^{(38)}$ The clinical use of paclitaxel-eluting devices was dramatically interrupted in November 2018 by the unexpected results of a meta-analysis including 28 RCTs with a total of 4432 patients. (39) The study described a 2-fold increase in all-cause mortality between 2 and 5 years of follow-up with paclitaxel-eluting DES/DCBs $(\mathrm{HR} 1.93,95 \% \mathrm{Cl} 1.27$ - 2.93), and a causal link between paclitaxel dose and mortality was hypothesised. These findings raised great concern, halted enrolment in RCTs on paclitaxeleluting devices, and prompted a worldwide call for high-quality data collection and analysis. Most recently, a confutation of the above-mentioned study came from a large analysis of German health claims data, investigating long-term mortality with paclitaxel-eluting devices from 2007 until the present in 6477 patients undergoing $107 \quad 112$ endovascular procedures. ${ }^{(40)}$ The use of paclitaxel-eluting devices was not associated with any signal of increased mortality up to 10 years of follow-up (Figure 4).

\section{VENOUSTHROMBOEMBOLISM}

In 2019, the ESC issued updated guidelines for management of patients with acute pulmonary embolism (PE). ${ }^{(41)}$ Key points include use of age-adjusted D-dimer cut-off in preclinical risk assessment. Furthermore, categorisation of PE events in "provoked" and "unprovoked" is no longer suggested. Rather, occurrence of index event in presence of "reversible risk factor", or in absence of any "identifiable risk factor" is suggested for patient stratification and guidance of treatment duration. For the first time, direct oral anticoagulants (DOACs) are recommended over vitamin $\mathrm{K}$ antagonists for $\mathrm{PE}$ treatment in eligible 
Safety analysis of paclitaxel based drug eluting devices for application peripheral arteries

Health claims data: 9.2 million insured people, 64 77I patients including 23 I37 DED

92 months median follow-up neither DCB nor DES associated with increased long-term mortality

$\mathrm{n}=6477 \mathrm{I}$ patients $\quad \mathrm{n}=20068 \mathrm{I}$ devices

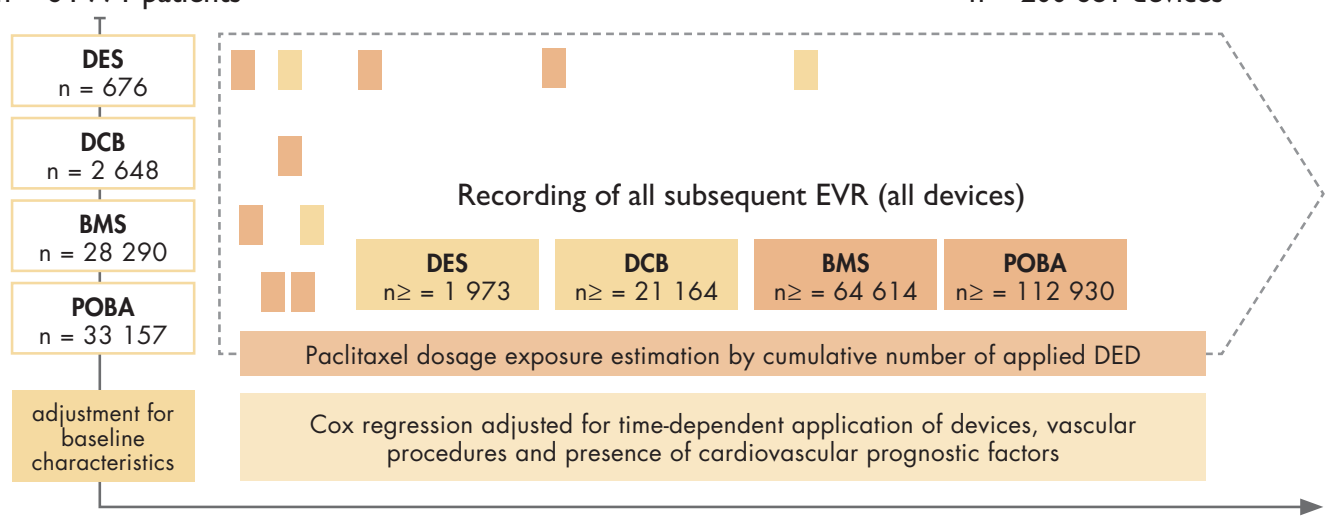

\section{Pre-phase}

24 months
Index hospitalisation

$01.01 .2007-31.12 .2015$
Follow-up until end of data registration 31.12.2107

Long-term mortality

\section{Stent EVR:}

1 year past stent EVR

2 years past stent EVR

3 years past stent EVR

4 years past stent EVR

5 years past stent EVR

6 years past stent EVR

7 years past stent EVR

8 until 11 years past stent EVR

Paclitaxel eluting effect:

1 year past DES

2 years past DES

3 years past DES

4 years past DES

5 years past DES

6 years past DES

7 years past DES

8 until 11 years past DES

Balloon EVR, any:

1 year past balloon EVR

2 years past balloon EVR

3 years past balloon EVR

4 years past balloon EVR

5 years past balloon EVR

6 years past balloon EVR

7 years past balloon EVR

8 until 11 years past balloon EVR

Paclitaxel coating effect:

1 year past $D C B$

2 years past $D C B$

3 years past $D C B$

4 years past DCB

5 years past DCB

6 years past $D C B$

7 years past $D C B$

8 until 11 years past DCB

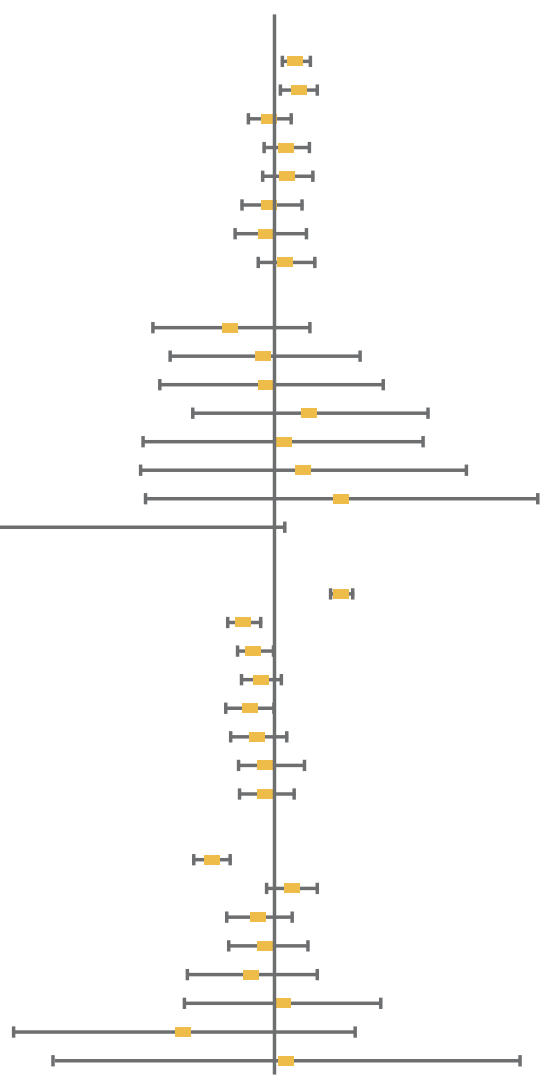

Extract from time-dependent Cox Regression Analysis

FIGURE 4: Long-term mortality after use of paclitaxel-based drug eluting devices (DED) in lower extremity arterial disease. A multivariable Cox regression analysis was developed including numerous comorbidities and all devices that were applied in each treated patient. For each distinct device, the analysis accounted for its type [drug-eluting stents (DES), drug-coated balloon (DCB), bare metal stents (BMS), and plain old balloon angioplasty (POBA)] and application date. The model also took concern of any non-constant time dependent effects: thus, a potentially detrimental effect of DED in the later course of time would become verifiable despite a potentially beneficial effect in the early years, or also any potential aggregation of subsequently applied devices. Combined hazard ratios for any scenario including multiple devices that were applied various years ago can be determined as the product of elementary hazard ratios. In summary, there was no signal that paclitaxel DCBs or DESs were associated with increased mortality up to I I years of follow-up. Reproduced with permission from ref. ${ }^{(40)}$ 
patients as for patients with atrial fibrillation. A reduced dose of apixaban or rivaroxaban for extended anticoagulation should be considered after the first 6 months of treatment. Edoxaban or rivaroxaban should be considered as an alternative to low molecular weight heparin in patients with non-gastrointestinal cancer who experience VTE. A new recommendation (class Ila, level A) proposes that carefully selected, low-risk PE patients should be considered for early discharge and home treatment, as long as proper outpatient care and anticoagulant therapy are possible (Figure 5). A recent prospective multicentre single-arm trial further corroborates this recommendation. Low-risk PE patients (no HESTIA criteria present, and absence of right ventricle enlargement/dysfunction) were early discharged (maximum of 2 nights in hospital) for home treat- ment with rivaroxaban. The study was prematurely terminated because of low symptomatic VTE recurrence and PE-related death rates (0.6\%; one-sided upper $99.6 \% \mathrm{Cl} 2.1 \%$ ), and low bleeding episodes (1.2\%) at 3 months from diagnosis. ${ }^{(43)}$ Careful selection of low-risk PE patients is key in successful home treatment; in this regard, clinical severity scores alone may not be sufficient to identify such a low-risk group, especially with regard to subclinical right ventricular dysfunction exclusion. Therefore, combining right ventricular assessment with clinical criteria further allows proper risk stratification as recently suggested by Barco, et al. ${ }^{(44)}$

The diagnosis of PE during pregnancy is challenging with wide pregnancy-related and PE suspicion symptoms overlapping. Overall, PE prevalence is, however, low thus exposing patients

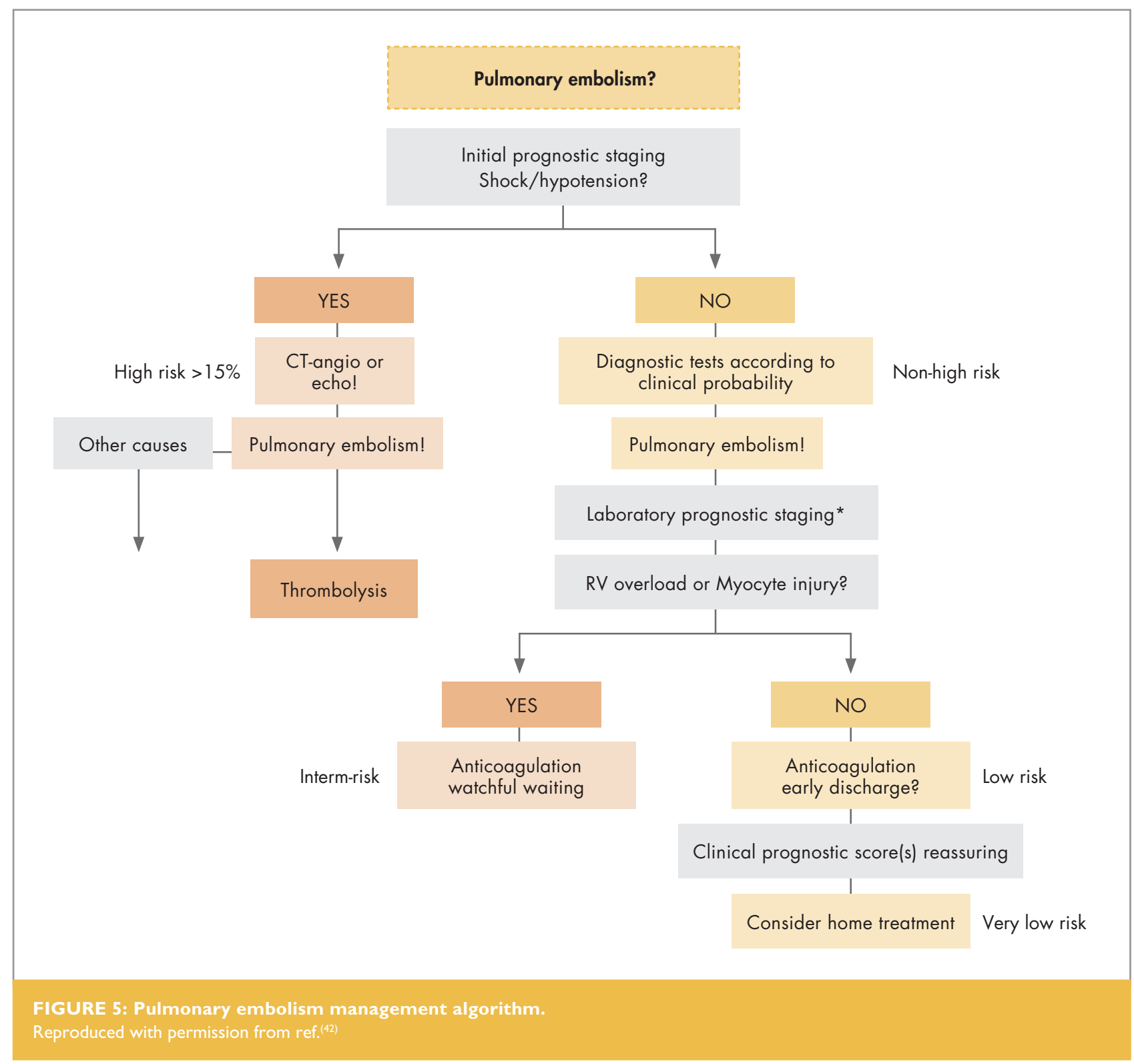


to unnecessary imaging tests. The 2019 ESC PE guidelines propose a dedicated diagnostic algorithm for suspected PE in pregnancy using stratification tools based on clinical presentation, D-Dimer testing, and compression ultrasonography of lower extremities. The pregnancy adapted YEARS algorithm, which takes into account these 3 parameters, was recently shown to safely rule out PE across all trimesters of pregnancy, avoiding a significant number of imaging tests. ${ }^{(45)}$

Management of vein thrombosis at unusual sites is challenging in practice. Whether patients with isolated distal deep vein thrombosis (IDDVT) should be systematically treated with anticoagulation is still questioned. It is suggested to stratify patients with IDDVT in high- and low-risk of recurrence. ${ }^{(46)}$ Those at high risk should be anticoagulated as for proximal deep vein thrombosis. ${ }^{(47)}$ With this regard, recent prospective registries suggested that patients with IDDVT may be treated with DOACs, although data from clinical trials are still missing. ${ }^{(48,49)}$

\section{PERSPECTIVES}

Exciting new scientific data published in 2019 shed more light on the nuances of atherosclerosis among the different peripheral vascular territories. The year 2020 is highly awaited for vascular specialists, with the completion of the Vascular Outcomes study of ASA along with rivaroxaban in endovascular or surgical limb revascularisation for peripheral artery disease (VOYAGER PAD) study, evaluating the efficacy and safety of rivaroxaban $2.5 \mathrm{mg}$ b.i.d. together with aspirin in reducing the risk of major thrombotic vascular events in subjects with symptomatic LEAD undergoing surgical or endovascular revascularisation (NCT02504216). Additionally, further data will become available addressing the safety of paclitaxel-coated technology for LEAD revascularisation. In the field of VTE, data from the CARAVAGGIO study (NCT03045406), comparing apixaban to dalteparin, for the treatment of acute VTE in cancer patients, are awaited.

Conflict of interest: C.H. reports research grants from Deutsche Forschungsgemeinschaft, Mitsubishi Cleansui, Wild Blueberry Association of North America, National Processed Raspberry Council, University of Surrey, Philips, Cranberry Institute, Rheacell; personal fees from Bayer, Novo Nordisk, Profil Institute outside the submitted work. J.J.F.B. reports grants and personal fees from Bayer and Rexgenero, personal fees from Amgen, outside the submitted work. M.D.C. reports personal fees from Bayer and Sanofi, outside the submitted work. H.R. reports grants from Bard, Biotronik, Bayer, personal fees from Diaplan, MedUpdate, Daichi Sankyo, and NeoVasc, personal fees and other support from NovoNordisk; other support from Bristol Myers Squibb and Pfizer; grants, personal fees, and other support from Pluristem outside the submitted work and is the senior author of a manuscript which is presented/reviewed in this article. A.P. reports personal fees from Actelion Pharmaceuticals Ltd and from Bristol-Myers Squibb Pharmaceuticals Limited, outside the submitted work. V.A. declares personal fees from Amgen, Bayer, Novartis, NovoNordisk, BMS/Pfizer alliance, and Sanofi, as speaker or advisor. L.M. and I.B. have declared no conflict of interest.

\section{REFERENCES}

I. De Carlo M, Mazzolai L, Bossone E, et al.; ESC Working Group on Aorta and Peripheral Vascular Diseases. The year in cardiology 2016: Peripheral circulation. Eur Heart J 2017;38:1028- 1033.

2. Aboyans V, Vrsalovic M, Madaric J, et al.; ESC Working Group on Aorta and Peripheral Vascular Diseases. The year 2018 in cardiology: Aorta and peripheral circulation. Eur Heart J 2019;40:872-879.

3. Aboyans V, Braekkan S, Mazzolai L, et al.; ESC Working Group on Aorta and Peripheral Vascular Diseases. The year 2017 in cardiology: Aorta and peripheral circulation. Eur Heart J 2018:39:730-738.

4. Aboyans V, Ricco JB, Bartelink MEL, et al.; ESC Scientific Document Group. 2017 ESC guidelines on the diagnosis and treatment of peripheral arterial diseases, in collaboration with the European Society for Vascular Surgery (ESVS): document covering atherosclerotic disease of extracranial carotid and vertebral, mesenteric, renal, upper and lower extremity arteries. Endorsed by: the European Stroke Organisation (ESO), The Task Force for the diagnosis and treatment of peripheral arterial diseases of the European Society of Cardiology (ESC) and of the European Society for Vascular Surgery (ESVS). Eur Heart J 2018:39:763-816.

5. Klarin D, Lynch J, Aragam K, et al. Genome-wide association study of peripheral artery disease in the Million Veteran Program. Nat Med 2019;25:1274-1279.

6. Leisegang MS, Bibli SI, Gunther S, et al. Pleiotropic effects of laminar flow and statins depend on the Kruppel-like factor-induced IncRNA MANTIS. Eur Heart J 2019;40:2523-2533.

7. Oppi S, Nusser-Stein S, Blyszczuk P, et al. Macrophage NCORI protects from atherosclerosis by repressing a pro-atherogenic PPAR $\gamma$ signature. Eur Heart J 2019;doi: 10.1093/eurheartj/ehz667.

8. Christersdottir T, Pirault J, Gistera A, et al. Prevention of radiotherapyinduced arterial inflammation by interleukin-I blockade. Eur Heart J 2019; 40:2495-2503.

9. Stiekema LCA, Stroes ESG, Verweij SL, et al. Persistent arterial wall inflammation in patients with elevated lipoprotein(a) despite strong low-density lipoprotein cholesterol reduction by proprotein convertase subtilisin/kexin type 9 antibody treatment. Eur Heart J 2019:40:2775-278I.

10. Mach F, Baigent C, Catapano AL, et al.; ESC Scientific Document Group. 2019 ESC/EAS Guidelines for the management of dyslipidaemias: Lipid modification to reduce cardiovascular risk. Eur Heart J 2019;doi:10.1093/ eurheartj/ehz455.

I I. Tzoulaki I, Castagne R, Boulange CL, et al. Serum metabolic signatures of coronary and carotid atherosclerosis and subsequent cardiovascular disease. Eur Heart J 2019:40:2883-2896.

12. Fernandez-Friera L, Fuster V, Lopez-Melgar B, et al. Vascular inflammation in subclinical atherosclerosis detected by hybrid PET/MRI. J Am Coll Cardiol 2019;73:1371-1382 


\section{REFERENCES}

13. Senders ML, Hernot S, Carlucci G, et al. Nanobody-facilitated multiparametric PET/MRI phenotyping of atherosclerosis. JACC Cardiovasc Imaging 2019; 12:2015-2026.

14. Charakida M, Georgiopoulos G, Dangardt F, et al. Early vascular damage from smoking and alcohol in teenage years: The ALSPAC study. Eur Heart 2019;40:345-353

15. Mortensen MB, Fuster V, Muntendam P, et al. Negative risk markers for cardiovascular events in the elderly. J Am Coll Cardiol 2019;74:I-I I.

16. Naslund $U, N g N$, Lundgren A, et al.; VIPVIZA trial group. Visualisation of asymptomatic atherosclerotic disease for optimum cardiovascular prevention (VIPVIZA): A pragmatic, open-label, randomised controlled trial. Lancet 2019;393:133-142.

17. Chiesa ST, Masi S, Shipley MJ, et al. Carotid artery wave intensity in mid- to late-life predicts cognitive decline: The Whitehall II study. Eur Heart J 2019:40:2300-2309

18. Amarenco P, Kim JS, Labreuche J, et al.; Treat Stroke to Target Investigators. A comparison of two LDL cholesterol targets after ischaemic stroke. N Eng J Med 20 I9;doi: 10. 1056/NEJMoal 9 | 0355 [Epub ahead of print].

19. Reddy VY, Neuzil P, de Potter T, et al. Permanent percutaneous carotid artery filter to prevent stroke in atrial fibrillation patients: The CAPTURE trial. J Am Coll Cardiol 2019;74:829-839.

20. Nazerian P, Mueller C, Vanni S, et al. Integration of transthoracic focused cardiac ultrasound in the diagnostic algorithm for suspected acute aortic syndromes. Eur Heart J 2019;40:1952-1960.

21. Erbel R, Aboyans V, Boileau C, et al.; ESC Committee for Practice Guidelines. 2014 ESC Guidelines on the diagnosis and treatment of aortic diseases: Document covering acute and chronic aortic diseases of the thoracic and abdominal aorta of the adult. The Task Force for the diagnosis and treatment of aortic diseases of the European Society of Cardiology (ESC). Eur Heart J 2014:35:2873-2926.

22. Ahn JM, Kim H, Kwon O, et al. Differential clinical features and long-term prognosis of acute aortic syndrome according to disease entity. Eur Heart J 2019:40:2727-2736.

23. Guala A, Teixidó-Tura G, Rodríguez-Palomares J, et al. Proximal aorta longitudinal strain predicts aortic root dilation rate and aortic events in Marfan syndrome. Eur Heart J 2019;40:2047-2055.

24. Desai $D$, Miranda $W$, Connolly $H$, et al. Flow dynamics in the false lumen in distal aorta following surgery for type A aortic dissection. Eur Heart 2019;40:561-561.

25. Chiu P, Goldstone AB, Schaffer JM, et al. Endovascular versus open repair of intact descending thoracic aortic aneurysms. J Am Coll Cardiol 2019, 73:643-651.

26. Lederle FA, Kyriakides TC, Stroupe KT, et al.; OVER Veterans Affairs Cooperative Study Group. Open versus endovascular repair of abdominal aortic aneurysm. N Engl J Med 2019;380:2126-2135.

27. Song P, Rudan D, Zhu Y, et al. Global, regional, and national prevalence and risk factors for peripheral artery disease in 2015: An updated systematic review and analysis. Lancet Glob Health 2019;7:el020-el030.

28. Beckman JA, Duncan MS, Damrauer SM, et al. Microvascular disease, peripheral artery disease, and amputation. Circulation 2019;140:449-458.

29. Hess $\mathrm{CN}$, Huang Z, Patel MR, et al. Acute limb ischemia in peripheral artery disease. Circulation 2019; 140:556-565.

30. Baumgartner I, Norgren L, Fowkes FGR, et al.; Executive Committee and Investigators of the EUCLID Trial. Cardiovascular outcomes after lower extremity endovascular or surgical revascularisation: The EUCLID trial. J Am Coll Cardiol 2018;72:1563-1572.

31. Navi BB, Reiner AS, Kamel H, et al. Arterial thromboembolic events preceding the diagnosis of cancer in older persons. Blood 2019;1 33:781-789.

32. Jukema JW, Szarek M, Zijlstra LE, et al.; ODYSSEY OUTCOMES Committees and Investigators. Alirocumab in patients with polyvascular disease and recent acute coronary syndrome: ODYSSEY OUTCOMES trial. J Am Col Cardiol 2019;74:1 167-1176.
33. Williams B, Mancia G, Spiering W, et al.; ESC Scientific Document Group. 2018 ESC/ESH Guidelines for the management of arterial hypertension. Eur Heart J 2018;39:3021-3104.

34. Lu Y, Ballew SH, Tanaka H, et al. 2017 ACC/AHA blood pressure classification and incident peripheral artery disease: the Atherosclerosis Risk in Communities (ARIC) study. Eur J Prev Cardiol 20 I9; doi: I 0.1 I 77/20474873 I 9865378 [Epub ahead of print].

35. McDermott MM, Kibbe MR, Guralnik JM, et al. Durability of benefits from supervised treadmill exercise in people with peripheral artery disease. J Am Heart Assoc 2019;8:e009380.

36. Lin E, Nguyen $\mathrm{CH}$, Thomas SG. Completion and adherence rates to exercise interventions in intermittent claudication: Traditional exercise versus alternative exercise: A systematic review. Eur J Prev Cardiol 2019;26:1625-1633.

37. Lin $J \mathrm{H}$, Brunson A, Romano PS, et al. Endovascular-first treatment is associated with improved amputation-free survival in patients with critical limb ischemia. Circ Cardiovasc Qual Outcomes 20 19; 12:e005273.

38. Laird JA, Schneider PA, Jaff MR, et al. Long-term clinical effectiveness of a drug-coated balloon for the treatment of femoropopliteal lesions. Circ Cardiovasc Interv 2019;12:e007702.

39. Katsanos K, Spiliopoulos S, Kitrou P, et al. Risk of death following application of paclitaxel-coated balloons and stents in the femoropopliteal artery of the leg: A systematic review and meta-analysis of randomised controlled trials. J Am Heart Assoc 2018;7:e0 I 245.

40. Freisinger E, Koeppe J, Gerss J, et al. Mortality after use of paclitaxel-based devices in peripheral arteries: A real-world safety analysis. Eur Heart J 2019; doi: 10.1093/eurheartj/ehz698.

4I. Konstantinides SV, Meyer G, Becattini C, et al.; ESC Scientific Document Group. 2019 ESC Guidelines for the diagnosis and management of acute pulmonary embolism developed in collaboration with the European Respiratory Society (ERS). Eur Heart J 2019;doi: 10.1093/eurheartj/ehz405.

42. Torbicki A. Assessing the severity of acute pulmonary embolism: Back to the future? Eur Heart J 2019;40:91 1-913.

43. Barco S, Schmidtmann I, Ageno W, et al. Early discharge and home treatment of patients with low-risk pulmonary embolism with the oral factor $\mathrm{Xa}$ inhibitor rivaroxaban: An international multicentre single-arm clinical trial. Eur Heart J 2019;doi: 10.1093/eurheartj/ehz367.

44. Barco S, Mahmoudpour SH, Planquette B, et al. Prognostic value of right ventricular dysfunction or elevated cardiac biomarkers in patients with lowrisk pulmonary embolism: A systematic review and meta-analysis. Eur Heart J 2019:40:902-910.

45. van der Pol LM, Tromeur C, Bistervels IM, et al. Pregnancy-adapted YEARS algorithm for diagnosis of suspected pulmonary embolism. N Engl J Med 2019;380:1 139-1149.

46. Valerio L, Ambaglio C, Barone M, et al. Recurrence risk after first symptomatic distal versus proximal deep vein thrombosis according to baseline risk factors. TH Open 2019;3:e58-e63.

47. Mazzolai L, Aboyans V, Ageno W, et al. Diagnosis and management of acute deep vein thrombosis: A joint consensus document from the European Society of Cardiology working groups of aorta and peripheral vascular diseases and pulmonary circulation and right ventricular function. Eur Heart J 2018;39:4208-4218.

48. Ageno W, Mantovani LG, Haas S, et al. Patient management strategies and long-term outcomes in isolated distal deep-vein thrombosis versus proximal deep-vein thrombosis: Findings from XALIA. TH Open 2019;3:e85-e93.

49. Schellong SM, Goldhaber SZ, Weitz Jl, et al. Isolated distal deep vein thrombosis: Perspectives from the GARFIELD-VTE registry. Thromb Haemost 2019;19:1675-1685. 